\title{
Microscopic study of electrical transport through individual molecules with metallic contacts: II. Effect of the interface structure
}

\author{
Yongqiang Xue * and Mark Ratner \\ Department of Chemistry and Materials Research Center, Northwestern University, Evanston, IL \\ 60208
}

(November 9, 2018)

\begin{abstract}
We investigate the effect on molecular transport due to the different structural aspects of metal-molecule interfaces. The example system chosen is the prototypical molecular device formed by sandwiching the phenyl dithiolate molecule (PDT) between two gold electrodes with different metal-molecule distance, atomic structure at the metallic surface, molecular adsorption geometry and with an additional hydrogen end atom. We find the dependence of the conductance on the metal-molecule interface structure is determined by the competition between the modified metal-molecule coupling and the corresponding modified energy level lineup at the molecular junction. Due to the close proximity of the HOMO of the isolated PDT molecule to the gold Fermi-level, this leads to the counterintuitive increase of conductance with increasing top metal-molecule distance that decreases only after the energy level line up saturates to that of the molecule chemisorbed on the substrate. We find that the effect on molecular transport from adding an apex atom onto the surface of a semi-infinite electrode is similar to that from increasing the metal-molecule distance. The similarity is reflected in both the charge and potential response of the molecular junction and consequently also in the
\end{abstract}


nonlinear transport characteristics. Changing the molecular adsorption geometry from a three-fold to an atop configuration leads to slightly favorable energy level lineup for the molecular junction at equilibrium and consequently larger conductance, but the overall transport characteristics remain qualitatively the same. The presence of an additional hydrogen end atom at the top metal-molecule contact substantially affects the electronic processes in the molecular junction due to the different nature of the molecular orbitals involved and the asymmetric device structure, which leads to reduced conductance and current. The results of the detailed microscopic calculation can all be understood qualitatively from the equilibrium energy level lineup and the knowledge of the voltage drop across the molecular junction at finite bias voltages.

85.65.+h,73.63.-b,73.40.-c

Typeset using REVTEX 


\section{INTRODUCTION}

Transport measurement of molecular junctions typically involves binding of molecules onto metallic electrodes through an appropriate end group. Often the contact with one of the electrodes is established by self-assembling the molecules on a single-crystal substrate. The other contact can be formed by vacuum deposition of a top metal layer, by using a scanning tunneling microscope (STM) tip or a conducting atomic force microscope $\operatorname{tip}^{1-8}$. Contacts to the electrodes can also be made by using atomic-scale break junctions ${ }^{9-13}$. The metal-molecule interface can therefore differ in metal-molecule distance, adsorption geometry and atomic structure of the metallic electrodes, or chemically in the types of the end groups and metals used. In most experiments, the structure of the metal-molecule interface is not known and cannot be controlled easily. This has hindered identifying the correct conduction mechanism through the molecular junction, since it is not clear whether the measured transport characteristics are intrinsic to the molecules or are due to features of the metal-moleule interface that might be non-reproducible from sample to sample ${ }^{12}$. The purpose of this second paper on microscopic study of single-molecule electronics is to elucidate the effect on molecular transport due to the different structural aspects of the metal-molecule interface. In the next paper, we discuss the effect on molecular transport due to the chemical aspects of the metal-molecule interface.

Since for a given molecule-metal combination, the atomic-scale structure of the metalmolecule interface differs in numerous ways, it is not useful to discuss exhaustively such differences and their effect on the transport characteristics without reference to specific transport measurements. Instead this work aims at identifying the key conceptual issues involved and demonstrates the use of such concepts through detailed microscopic study of selected aspects of the metal-molecule interface. Such concepts should then be useful in giving a clear indication of whether given device characteristics originate from features of

the metal-molecule interface structure. In the first paper of this series ${ }^{16}$ (refereed to as $\mathbf{I}$ in this work), we have identified two key factors for understanding the transport character- 
istics of a molecular junction: the equilibrium energy-level lineup and the nonequilibrium charge/potential response to the applied bias. Electronic processes at the metal-molecule interface play different roles in determining these two factors: (1) At equilibrium, the symmetry and the magnitude of the metal-molecule orbital overlap determine the capabilities of the molecular states to function as effective conduction channels, while the coupling-induced charge and potential perturbation determine the shift of the molecular level relative to the metal Fermi-level; (2) Out of equilibrium, the asymmetry of the coupling at the sourcemolecule and drain-molecule interfaces determines the net charge flow into the molecule, but the spatial distributions of the charge response and the voltage drop are determined by the potential landscape across the entire molecular junction. At not too high bias voltage, this affects mainly the shift of the molecular levels with bias voltage (see I). Our discussion will therefore focus on analyzing how the different aspects of the interface structure affect the above two types of electronic processes.

Since the effect of the metal-molecule interface structure on the device characteristics is seen most clearly for symmetric molecules, we will use as example the prototypical molecular device formed by attaching the phenyl dithiol molecule (PDT) onto two gold electrodes through the end sulfur atoms. Since in many experiments on molecular transport, the molecules are self-assembled on a single-crystal substrate and the structure of the moleculesubstrate contact can be considered well-defined, we start from the reference interfacial configuration where the molecules form symmetric contact with two semi-infinite gold $<111>$ electrodes (see $\mathbf{I}$ ) and investigate the change in its transport characteristics due to structural differences in terms of the metal-molecule distance, atomic structures of the metallic surfaces and the adsorption geometry. Since in practice it is not clear whether the end hydrogen atoms are desorbed upon electrode contact $^{14,15}$, we will also investigate the effect on current transport due to an additional end hydrogen atom at the top metal-molecule interface. The theoretical approach we use in elucidating the effect of the metal-molecule interface structure were discussed in detail in $\mathbf{I}$ and also elsewhere ${ }^{17,18}$. We use the same modeling methodology as described in I. In particular, we have used the BPW91-parameterization 
of the spin-density-functional theory ${ }^{19^{-21}}$ and the $a b$ initio pseudopotential ${ }^{22}$ with the corresponding energy-optimized gaussian basis $\operatorname{sets}^{23,24}$. The calculation is performed using a modified version of GAUSSIAN98 ${ }^{25}$. We will focus on the results of the computation and the conceptual understanding derived from them (we use atomic units throughout the paper unless otherwise noted).

\section{THE EFFECT OF THE METAL-MOLECULE DISTANCE}

In the reference device structure, the molecule forms symmetric contact with two semiinfinite gold $<111>$ electrodes, and the molecule sits on top of the center of the triangular gold pad (see I). The end sulfur atom-metal surface distance at both interfaces is $1.9(\AA)$. We first consider the effect on current transport from increasing the metal-molecule distance for the right contact (denoted top contact from here on. The left contact is denoted the substrate contact). Increasing the top metal-molecule distance reduces the strength of orbital coupling across the interface without changing its symmetry, which also affects the equilibrium energy-level lineup since it changes the magnitude of the charge and potential perturbation across the molecular junction. The conductance of the metal-molecule-metal junction will depend on the competition between these two factors. This is demonstrated by examining both the equilibrium transmission versus energy (TE) characteristics and the projected molecular density of states (PDOS). The calculated TE characteristics, the PDOS and the current/conductance-voltage characteristics of the molecular junction for symmetric contact case and for cases where the top metal-molecule distance increased by $\Delta L=0.5,1.0,1.5,2.0(\stackrel{\AA}{A})$ are shown in Figs. (1-3). To check that the calculation is not sensitive to small changes in the metal-molecule distance, we have also calculated the transport characteristics of the molecular junction for $\Delta L=0.1,0.2(\AA)$, which show smooth deviation from the reference symmetric contact case.

Increasing the top metal-molecule distance up to $1(\stackrel{\circ}{A})$ increases the conductance of the

molecular junction. Although this result is counterintuitive, it is readily understood by 
examining the energy-level lineup at the metal-molecule-metal junction. The calculated HOMO level of the isolated PDT molecule in the spin-singlet state (with the end hydrogen atoms removed) is $-5.33(\mathrm{eV})$, very close to the metal Fermi-level $-5.31(\mathrm{eV})$. For the symmetric contact case, the charge transfer and the associated electrostatic potential change across the interface push it down to about $1(\mathrm{eV})$ below the Fermi-level (deduced from the corresponding peak positions in the PDOS plot, see I). Increasing the top metal-molecule distance will therefore move the HOMO up to closer alignment with the metal Fermi-level, which overcompensates the decreased coupling strength in determining the transmission coefficient at the Fermi-level and correspondingly the low-bias conductance. This trend continues as we increase $\Delta L$ from 0 to $1.0(\AA)$. Note that although the transmission coefficient at the Fermi-level increases, the transmission through the middle of the HOMO-LUMO gap decreases monotonically with increase of the top metal-molecule distance as expected. We can also see that the effect on the alignment of the LUMO level is much weaker than that of the HOMO as we increase $\Delta L$ from $0.5(\stackrel{\circ}{A})$ to $1.0(\AA)$, since the electron distribution of the LUMO is localized in the interior of the molecule (see I). As we further increase the top metalmolecule distance to $\Delta L=1.5(\stackrel{\circ}{A})$, the coupling across the top metal-molecule interface becomes so weak such that its effect on the energy level lineup saturates, i.e., the energy level lineup approaches that of the molecule chemisorbed on the substrate. Further increasing the top metal-molecule distance will then reduce the transmission coefficient and the low-bias

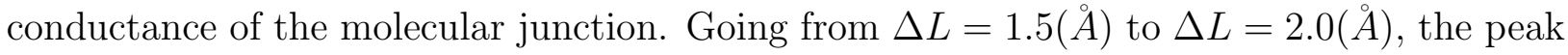
positions in both TE and PDOS plots corresponding to the HOMO and LUMO level do not change much, but the transmission coefficient is reduced across the entire energy spectrum (Fig. (2)).

As the top metal-molecule distance increases, the equilibrium transmission characteristics also change substantially. In particular, the double-peak structure corresponding to resonant transmission through the LUMO and $\mathrm{LUMO}+1$ is reduced to a single peak for transmission through the LUMO+1 state. The transmission probability away from the two peaks at $\mathrm{HOMO}$ and $\mathrm{LUMO}+1$ is reduced rapidly with the increasing top metal-molecule distance. 
Examinations of the corresponding LDOS show that the LUMO is mainly localized on the peripheral hydrogen atoms, which leads to negligible orbital overlap with the top metal states and rapid reduction of the transmission probablilty with increasing top metal-molecule distance. The HOMO and LUMO+1 levels instead have large weights on both end sulfur atoms, so their transmission probability decreases much slower with the increasing top metalmolecule distance. Since transport is dominated by by tunneling through HOMO in the bias voltages studied here, the changes in the transmission through the unoccupied molecular states do not affect the device characteristics.

As $\Delta L$ increases, the asymmetry of the $\mathrm{I}-\mathrm{V}$ and $\mathrm{G}-\mathrm{V}$ characteristics with respect to bias polarity also increases (Fig. (3)), since the bias-induced modification of molecular states (the Stark effect) differes at different bias polarities due to the asymmetry (the bias polarity is chosen such that positive bias voltage corresponds to electron injection from the top contact). This is clear from the bias-dependence of the molecular levels in Fig. (4). At large top metal-molecule distance, the molecule couples strongly to the substrate metal but very weakly to the top metal. The calculated I-V and G-V characteristics (Fig. (3)) correspond to that obtained for tunneling through a chemisorbed molecule, e.g., measured using a STM tip with a large vacuum gap ${ }^{26}$. At first sight, this suggests that the shift of the molecular levels with applied bias voltage should follow that of the electrochemical potential of the substrate contact. However, this is only true at positive bias voltages. At negative bias voltage, they may deviate from each other as a function of the metal-molecule distance. This can only be explained from an atomi-level analysis of the charge and potential response of the molecule to the applied bias voltage.

The spatial distribution of the charge perturbation and the voltage drop across the molecular junction for $\Delta L=1.5(\stackrel{\circ}{A})$ at voltages of $V=2.0(V)$ and $-2.0(V)$ are shown in Fig. (5). The spatial distribution of the charge perturbation is obtained by integrating the difference in electron density at finite and zero biases along the z-axis and plotted as a function of position in the xy-plane (defined by the benzene ring). The voltage drop is obtained by evaluating the difference between the electrostatic potential at finite and zero 
biases, which obeys the boundary condition of approaching $-V / 2(V / 2)$ at the substrate (top) electrode. Due to the increased metal-molecule distance at the top contact, a larger potential barrier is created at the top metal-molecule interface than that at the substratemolecule interface, and it is easier for the electrons to move from the top contact side to the substrate side than the other way around (not shown here). This leads to distinct behaviors in the molecular response to the applied bias at different bias polarities.

At $V=2.0(V)$, electrons are injected into the molecule from the top contact, which forms the bottleneck for transport. The direction of the applied field favors the electron flow within the molecules. Most of the charge perturbation therefore occurs at the top metal-molecule interface, with a large decrease in the electron density on the molecule side of the top metalmolecule interface (Fig. (5)). Since electrons on the substrate side of the junction can move freely to screen effectively the applied field, most of the voltage drops across the top metalmolecule interface. At $V=-2.0(V)$, electrons are injected from the substrate contact across a smaller barrier. The electrons cannot move freely to screen the applied field compared to the positive bias case. Correspondingly, the charge perturbation on the substrate side of the molecular junction is significant and significant voltage drop occurs before reaching the top metal-molecule interface. As a result, at large top metal-molecule distance, the molecular level shift follows that of the substrate electrode at positive bias voltage, but shows more complicated pattern for negative bias voltage with larger effect on the occupied molecular states. The total number of electrons within the molecule decreases with increasing positive bias voltage since it is easier to extract electrons from the molecule through the substrate contact than to inject electron into the molecule through the top contact. But the total number of electrons is approximately constant at negative bias polarity. This gives rise to the large asymmetry in $\mathrm{I}-\mathrm{V}$ and $\mathrm{G}-\mathrm{V}$ characteristics with respect to the bias polarity.

We can also examine more directly the asymmetry in the device characteristics from the voltage dependence of the transmission (TE) characteristics at $\Delta L=1.5(\AA)$ (not shown here). The conductance reaches its peak when one of the metal Fermi-levels moves into alignment with the peak positions in the TE characteristics. We find that at large top 
metal-molecule distance, the conductance reaches its peak near zeros bias in the negative bias direction as the right metal Fermi-level moves down passing the HOMO level. At positive bias voltage, the G-V characteristics probes the states in the HOMO-LUMO gap resulting in a much reduced conductance.

\section{THE EFFECT OF THE ATOMIC STRUCTURE OF THE METALLIC SURFACE}

If the transport through the molecule is measured using a scanning tunneling microscope (STM) tip, it would not be appropriate to model the top contact as a semi-infinite crystal since atomic-scale structures may exist at the tip apex. If the current is measured using atomic-size break junctions, both contacts may include atomic-scale structures on their surface. In this section, we investigate the effect on current transport due to the presence of atomic structures on the metallic surface. We consider two simple device models: For model 1, we consider the top electrode as composed of one apex atom sitting on top of the triangular gold pad of the semi-infinite $<111>$ crystal; for model 2 , we consider both electrodes as composed of one apex atom sitting on top of the triangular gold pad of the semi-infinite $<111>$ crystal.

The equilibrium energy-level line up (as reflected in the TE and PDOS plots) for both models are shown in Fig. (7). The calculated I-V and G-V characteristics are shown in Fig. (8). We find remarkable similarity between adding a apex atom onto the top electrode (model 1) and increasing the top metal-molecule distance in determining the equilibrium "band" lineup as well as the nonlinear transport characteristics. Both cases result in the HOMO level being closer to alignment with the metal Fermi-level. Both cases also show a similar lineup scheme for the LUMO level. Note that the device characteristics for model 1 show similar feature with that of $\Delta L=1.0(\AA)$ in sec. II, although the distance between the end sulfur atom and the semi-inifinite crystal surface here would correspond to $\Delta L=2.3(\AA)$. A simple

explanation of the electronic origin of this similarity is as follows: the valence orbital of an 
isolated gold apex atom is of $s$ type which has minimal overlap with the sulfur $\pi$ orbitals on the molecule. The hybridization between the apex gold atomic orbitals and the gold surface states introduces non- $s$ type symmetry, so the coupling between the apex gold atom and the sulfur end atom is stronger than that would be obtained for an isolated gold-sulfur bond, but is much weaker than that of the reference interfacial configuration. Although this has been pointed out before ${ }^{27,28}$, further insight into the problem can only be obtained through an atomic-level analysis of junction charge and potential response because the simple bonding analysis cannot explain the similarities in the nonlinear transport characteristics.

The potential perturbation upon formation of the molecular junction (at equilibrium) for model 1 is shown in Fig. (9), while the charge and potential response for bias voltages of $-2.0(\mathrm{~V})$ and $2.0(\mathrm{~V})$ are shown in Fig. (10), where we have also shown the position of the molecule and the gold apex atom. Compared to the molecule-substrate contact, the electron density of the equilibrium junction decreases in the $p_{x}$ orbital region of the top apex atom, since the gold apex $p_{x}$ orbital cannot form a bond with the end sulfur atom but is involved in the bonding between the apex atom and the top surface atoms (not shown here). This difference in the charge perturbation at the two interfaces leads to asymmetry in the potential for the equilibrium junction. Although the potential change within the molecule favors electron flow from the substrate side to the top contact side, the barrier at the apex atom-molecule interface is still larger than that at the substrate-molecule interface. This leads to similar charge and potential response to the applied bias voltages as those described in the previous section where the top metal-molecule distance is increased. At positive bias, the voltage drop across the molecular junction occurs mostly at the right metal-molecule contact, but at negative bias voltage, a significant amount of the voltage drop also occurs within the molecule (Fig. (10)). Compared to the case of increasing top contact-molecule distance, the amount of the voltage drop within the molecule core is slightly larger here due to the less favorable potential landscape for electron flow within the molecule core.

The similar charge and potential response also leads to similar molecular level shift at different bias polarities (not shown here). Note that voltage also drops from the apex atom 
to the bulk of the electrode (the voltage should approach $+(-) 1.0(V)$ as we approach the bulk of the electrode). This is because a single gold atom at the apex cannot effectively screen the applied field, which behaves more like the other atoms in the molecule. This may have important implications in transport measurement using STM tips, since sharp atomicscale structures and correspondingly localized electron states can develop at the apex of the metallic tip especially for transition metals ${ }^{29}$. If a significant amount of the voltage drops from the apex to the metal bulk, negative differential resistance can be observed for single molecules ${ }^{5,30,31}$.

For junction model 2, the above analysis of charge and potential response at the top metal-molecule contact applies also to the substrate-molecule contact. Compared to the reference symmetric contact configuration, the contact to the electrodes leads only to potential barrier at the apex atom-end sulfur interface. The potential landscape within the molecule core is rather flat, while for the reference contact configuration there is an additional potential barrier across the sulfur-benzene bonding region (see $\mathbf{I}$ ). As a result, once electrons are injected into the molecule, it would be easier for them to be extracted through the other contact. This leads to different charge and potential response at finite applied bias: most of the charge perturbation and voltage drop occurs at the electron injecting side of the molecular junction, yielding results (not shown here) very similar to Fig. (8). As a result, the direction of the molecular level shift with applied bias follows that of the Fermi-level of the electron injecting contact at both bias polarities.

The similarity between junction model 1 and increasing top metal-molecule distance by $\Delta L=1.0(\AA)$ is reflected equally in the equilibrium transmission characteristics and the corresponding LDOS plot, where both the magnitude and the energy-dependence of the transmission coefficient as well as the charge distribution of the contact-perturbed molecular states show similar behavior. By contrast, in the equilibrium transmission characteristics of the junction model 2, the overall energy-dependence of the transmission coefficient corresponds closely to the reference contact configuration being shifted up to move into closer resonance with the metal Fermi-level due to the weaker bonding across both metal-molecule 
interfaces. But similar to junction model 1, the double-peak structure corresponding to transmission through the LUMO and LUMO+1 has been reduced to a single peak. Compared to junction model 1, there is a sharp peak in the TE characteristics through the metal-induced-gap states at $E=-3.1(\mathrm{eV})$ in the HOMO-LUMO gap. The TE through the occupied molecular states are also much larger than that of junction model 1, and sharper than the reference configuration. All these follow from the symmetry and magnitude of the charge distribution of the corresponding molecular states (determined from the LDOS). The charge distribution (the LDOS) of the LUMO and LUMO+1 levels remain qualitatively the same for the reference configuration, increasing top metal-molecule distance, junction model 1 and model 2. Insertion of an apex atom as well as increasing the metal-molecule distance results in much reduced overlap between the LUMO state and the metal surface states leading to negligible transmission. The LUMO+1 state also shows similar charge distribution in all cases, with large weight on both end sulfur atoms. This leads to large TE which are not affected as strongly by inserting an apex atom or increasing metal-molecule distance as other states. The larger TE through the HOMO-LUMO gap and the occupied molecular states for junction model 2 is instead due to the symmetry of the junction configuration. For junction model 2, the corresponding LDOS shows equally large charge distribution at both metal-molecule interfaces, while for junction model 1, they show much reduced charge at the subtrate-molecule contact. The peaks for model 1 and 2 are sharper than the reference configuration because the states are more localized. So the transmission through the occupied states is reduced by inserting an apex atom at one contact but remains large when apex atoms are inserted at both contacts.

To summarize, the results shown here highlight the important effect that atomic-scale electrode structures may have on molecular transport characteristics. 


\section{THE EFFECT OF THE MOLECULAR ADSORPTION GEOMETRY}

In computational study of molecular junctions, the adsorption geometry is often chosen with the sulfur atom in a three-fold site above the electrode surface. In this section, we consider a different adsorption geometry with the sulfur end atom atop one surface gold atom. Each surface gold atom has 6 nearest-neighbors, therefore we include 7 gold atoms on each metallic surface into the "extended molecule" (denoted model 3). We also consider the effect on current transport when the top metal-molecule distance is increased by $1.0(\AA)$ (denoted model 4) for comparison with the results in sec. II.

For device model 3, the coupling between the molecule and the electrodes is reduced due to the less favorable orbital overlap between the end sulfur atom and the gold surface atom directly underneath it. But the reduction is smaller than that in sec. III due to the molecular orbital overlap with the other 6 gold atoms on the surface. Compared to the case of threefold adsorption, the magnitude of the charge transfer and the potential perturbation are smaller (not shown here). As a result, the HOMO level is moved slightly closer to the metal Fermi-level for device at equilibrium (Fig. 11), similar to the effect of slightly increasing the metal-molecule distance. This geometry effects the molecular states differently depending on their composition. For sulfur based states like HOMO, the PDOS is narrowed due to the reduced mixing with the metal surface states. For carbon based states like LUMO, the PDOS is broadened since the mixing with the metal surface states is stronger due to the proximity of more gold surface atoms. The same consideration leads to the more broadened PDOS towards the HOMO-LUMO gap for HOMO and LUMO. Also notable is that the once pronounced transmission peak for tunneling through the metal-induced-gap-states is suppressed since the mixing through the end sulfur atom is suppressed. The trend continues as the top metal-molecule distance is increased (model 4 in Fig. 11). Again increasing the top metal-molecule distance leads to the favorable alignment of the HOMO and increases the zero-bias conductance (Fig. 12).

Due to the broadened density of states in the HOMO-LUMO gap and more favorable 
equilibrium energy level lineup, the low-bias conductance is higher and its increase with bias voltage is less steep in the atop adsorption geometry (Fig. 12). The peak positions in the G-V characteristics are reached at slightly smaller bias voltage due to the smaller difference between the HOMO level and the equilibrium Fermi-level.

\section{THE EFFECT OF A RETAINED END HYDROGEN ATOM}

For the thiol molecules self-assembled on a gold substrate, it is commonly believed that the end hydrogen atom is desorbed during the final stage of the self-assembly process ${ }^{14,15}$. But it is not clear whether the hydrogen atom at the top contact is desorbed after the formation of a stable contact since different experimental techniques have been used. In this section we investigate whether the presence of an additional end hydrogen atom at the top contact affects significantly the transport characteristics. The structure of the molecular radical is obtained first by optimizing the geometry of the molecule with both end $\mathrm{H}$ atoms at the BPW91/6 - 31G* level and then removing the $\mathrm{H}$ atom at the substrate side, forming a molecular spin doublet (optimizing the geometry of the molecular radical directly gives similar results). The hydrogen atom should inhibit the coupling between the sulfur atom

and the top metal surface. For comparison with the results in sec. II, we assume the same three-fold sulfur-gold adsorption geometry but with increased sulfur-surface distance of $2.2(\AA)$ (the hydrogen-surface distance is $2.0(\AA))$.

The H-atom addition increases the number of electrons of the molecule by one. For this doublet system, the three molecular orbitals energetically closest to metal Fermi-level (corresponding to the HOMO-1, HOMO and LUMO) for electrons with different spin differ, as shown in Fig. (13), which are also different from those of the molecule with both end $\mathrm{H}$ atoms removed. The $\mathrm{H}$ addition breaks the symmetry of the molecular orbitals. Both the HOMO for spin-up electron and the HOMO-1 for spin-down electron have large weight only on the left (substrate) sulfur and therefore can couple strongly only to the left (substrate) contact. On the other hand, both the HOMO-1 for spin-up electron and the HOMO for spin- 
down electron show electrons delocalized throughout the molecule, with larger weight on the left sulfur for the spin-down electron. The LUMO for both spin-up and spin-down electrons shows large weight only on the interior carbon atoms, not affected by the $\mathrm{H}$ addition.

The $\mathrm{H}$ addition at the top (right) metal-molecule interface saturates the sulfur $\pi$ bond and reduces significantly the amount of charge transfer into the top sulfur atom upon adsorption onto the electrodes due to the saturated bonding. The decreased molecule-metal bonding leads to a larger potential barrier at the top interface. Indeed, the magnitude of the charge transfer at the top contact is smaller than that obtained in sec. II for the case of $\Delta L=2(\AA)$, which corresponds to a sulfur-surface distance of $3.9(\stackrel{\circ}{A})$. This leads to quite different energylevel lineup scheme for the molecular junction at equilibrium, as shown in Fig. (14) where we plot TE and the PDOS in the molecule. The peak positions corresponding to transmission through the HOMO and LUMO levels are lowered relative to the reference structure, giving a more symmetric location of the metal Fermi-level, although the HOMO level is still closer to the Fermi level than the LUMO.

Note that although both the energy and the electron distribution associated with the molecular states depend on the spin direction for the isolated molecule, the transmission coefficient and the PDOS in the molecular junction are identical for both spin directions once the self-consistent calculation is converged. The reason is as follows: For the strong moleculemetal coupling regime we consider here, the open-shell PDT molecular radical (with one $\mathrm{H}$ end) within the molecular junction is only part of a large quantum system. Since the filling of the electron states is determined by the Fermi-distribution of the semi-infinite electrodes which are non-magnetic, there is no physical origin for breaking the spin symmetry of the system. The PDT molecular radical in contact with two gold electrodes behaves in much the same way as an open-shell atom within a closed-shell molecule since the electron states are delocalized across the molecular junction. The situation will be dramatically different in the Coulomb blockade regime when the molecule is weakly coupled to both electrodes or when a high spin-degeneracy atom is inserted into the molecule ${ }^{10,11}$, in which cases a spin-dependent interaction term needs to be included in the Hamiltonian describing the molecular junction 
and spin-dependent electron scattering can dominant especially at low temperature.

Interestingly, although the addition of a $\mathrm{H}$ atom moves the frontier orbitals closer to the metal Fermi-level and to each other (Fig. (13)), the transmission characteristics of the equilibrium junction remain qualitatively the same as for the singlet molecular biradical besides the shift in the peak positions(Fig. (14)). The corresponding LDOS shows that the charge distributions associated with both the HOMO and LUMO levels remain similar in shape with the addition of the end $\mathrm{H}$ atom. The transmission coefficient at the Fermi level is slightly reduced, but the overall transmission characteristics in the HOMO-LUMO gap are similar in both cases. The main effect of introducing the end $\mathrm{H}$ atom has been in creating a nonsymmetric device structure.

The calculated I-V and G-V characteristics are shown in Fig. (15). Both the current and the conductance are reduced by the presence of the hydrogen within the bias range studied. Substantial asymmetry with respect to the bias polarity is introduced due to the the different contact configuration. Similar to the results discussed in previous sections, peak in the conductance is reached only at negative bias polarity due to the larger voltage drop at the top metal-molecule contact at positive bias (not shown here). This can be seen clearly from the bias-dependence of the transmission characteristics in Fig. (16). Applying a negative bias decreases the difference between the HOMO level position of the molecular radical (one $\mathrm{H}$ end) and the molecular biradical, leading to the same bias voltage of $V=-1.6(V)$ where conductance reaches its peak (the peak position in the transmission characteristics coincides with the fermi level of the top contact). The shift with applied bias of the LUMO level is much larger than that of the HOMO, but since the conductance is again determined mainly by the HOMO states, this doesn't affect the calculated transport characteristics.

\section{CONCLUSIONS}

We have investigated the effect on molecular transport due to different structural aspects

of the metal-molecule interface. For a given metal-molecule combination, the differences in 
the interface structure not only lead to different metal-molecule coupling, but also to different energy-level lineup and to different electrostatic potential profile across the the molecular junction. The difference in the resulting nonlinear transport characteristics reflects both the difference in the energy-level lineup scheme as well as the difference in the potential response of the molecular junction.

These considerations are illustrated through detailed microscopic calculation of the prototypical molecular device formed by sandwiching the PDT molecule between two gold electrodes. The metal-molecule interface structures investigated differ in metal-molecule distance, atomic structures at the metal surface, adsoprtion geometry and the presence of an additional end hydrogen atom. The chosen system is representative of the current experimental work on molecule electronics, but is also unique in that the HOMO level of the isolated PDT molecule is very close to the gold Fermi-level. This leads to a counterintuitive increase of conductance with increasing top metal-molecule distance beacuse the reduced coupling leads to closer alignment of the HOMO level with the gold Fermi-level. The conductance decreases only after passing the maximum metal-molecule distance where the energy level lineup becomes essentially identical to that of the molecule chemisorbed on the substrate. For the gold-PDT-gold junction we consider here this happens around $\Delta L=1.5(\AA)$ corresponding to the sulfur-top metal distance of $3.4(\AA)$. Adding one apex atom onto the semi-infinite surface of the bulk electrodes is equivalent in its effects to the increase of the metal-molecule distance due to the unfavorable orbital overlap with the sulfur end atom. The similarity is reflected in the contact-perturbed molecular states, the charge and potential response of the molecular junction to the applied bias and the nonlinear transport characteristics. Changing to an atop molecular adsorption geometry leads to slightly favorable energy level lineup for the molecular junction at equilibrium and consequently larger conductance, but the overall transport characteristics remain qualitatively the same. The presence of the additional hydrogen end atom at the top metal-molecule contact affects substantially the electronic processes in the molecular junction due to the different nature of the molecular orbitals and the asymmetric device structure involved, reducing the 
conductance and current compared to the case where the end hydrogen atom is desorbed during the formation of the contact. The results of the microscopic calculation can all be understood from the equilibrium energy-level lineup combined with qualitative knowledge of the voltage drop due to the asymmetry in the two metal-molecule contacts. Since the equilibrium energy level lineup is quite sensitive to the atomic-scale structures of the metalmolecule interface, a correct identification of the device structure is essential for explaining the transport characteristics of any molecular junction.

For different metal-molecule combinations, the lineup of the molecular level with respect to the metal surface band, and therefore the functional dependence of transport characteristics on interface structure will be different. In particular, both the energy level lineup and the metal-molcule coupling can be modified by simply replacing the end contact atom (sulfur in this paper) with other chemical groups without changing the metal and the molecule core. This and other chemical aspects of the metal-molecule interface are interesting subjects which deserve further analysis.

\section{ACKNOWLEDGMENTS}

We thanks A. Nitzan, S. Datta, V. Mujica and H. Basch for useful discussions. This

work was supported by the DARPA Molectronics program, the DoD-DURINT program and the NSF Nanotechnology Initiative. 


\section{REFERENCES}

*Author to whom correspondence should be addressed. Email: ayxue@chem.nwu.edu

${ }^{1}$ C. Joachim, J. K. Gimzewski and A. Aviram, Nature 408, 541 (2000) and references thererein.

${ }^{2}$ R. M. Metzger et al. , J. Am. Chem. Soc. 119, 10455 (1997); R. M. Metzger, Acc. Chem. Res. 32, 950 (1999).

${ }^{3}$ J. Chen, M. A. Reed, A. M. Rawlett and J. M. Tour, Science 286, 1550 (1999).

${ }^{4}$ S. Datta, W. Tian, S. Hong, R. Reifenberger, J. J. Henderson and C. P. Kubiak, Phys. Rev. Lett. 79, 2530 (1997).

${ }^{5}$ Y. Xue, S. Datta, W. Tian, S. Hong, R. Reifenberger, J. J. Henderson and C. P. Kubiak, Phys. Rev. B. 59, 7852 (1999).

${ }^{6}$ S. Hong, R. Reifenberger, W. Tian, S. Datta, J. Henderson and C. P. Kubiak, Superlatt. Microstruc. 28, 289 (2000).

${ }^{7}$ N. B. Zhitenov, H. Meng and Z. Bao, Phys. Rev. Lett. 88, 226801 (2002).

${ }^{8}$ X.D. Cui et al. , Science 294, 571 (2001).

${ }^{9}$ M. A. Reed, C. Zhou, C. J. Muller, T. P. Burgin and J. M. Tour, Science 278, 252 (1997); Mark A. Reed, Proc. IEEE 97, 652 (1999) and references therein.

${ }^{10}$ J. Park et al. , Nature 417, 722 (2002).

${ }^{11}$ W. Liang et al. , Nature 417, 725 (2002).

${ }^{12}$ J. Reichert et al. , Phys. Rev. Lett. 88, 176804 (2002).

${ }^{13}$ J. G. Kushmerick et al. , Phys. Rev. Lett. 89, 86802 (2002).

${ }^{14}$ Y. Xia and G.M. Whitesides, Adv. Mater. 7, 471 (1995). 
${ }^{15}$ M.T. Cygan et al. , J. Am. Chem. Soc. 120, 2721 (1998).

${ }^{16}$ Y. Xue and M. A. Ratner, previous paper.

17 Y. Xue, S. Datta and M. A. Ratner, J. Chem. Phys. 115, 4292 (2001).

${ }^{18}$ Y. Xue, S. Datta and M. A. Ratner, Chem. Phys. 281, 151 (2002). (LANL archive: xxx.lanl.gov/cond-mat/0112136).

${ }^{19}$ A. D. Becke, Phys. Rev. A. 38, 3098 (1988).

${ }^{20}$ J. P. Perdew and Y. Wang, Phys. Rev. B. 38, 12228 (1988); M. Ernzerhof, J. P. Perdew and K. Burke, in Density Functional Theory I, edited by R. F. Nalewajski (Springer, Berlin, 1996).

${ }^{21}$ O. Gunnarson and B. I. Lundqvist, Phys. Rev. B 13, 4274 (1976); R. O. Jones and O. Gunnarson, Rev. Mod. Phys. 61, 689 (1989).

${ }^{22}$ L. R. Kahn, P. Baybutt and D. G. Truhlar, J. Chem. Phys. 65, 3826 (1976).

${ }^{23}$ For carbon and sulphur, we use the pseudopotential and the corresponding polarized split valence basis sets of W. J. Stevens, H. Basch and M. Krauss, J. Chem. Phys. 81, 6026 (1984); For gold, we use the pseudopotential and the valence basis sets of P. J. Hay and W. R. Wadt, J. Chem. Phys. 82, 270 (1985).

${ }^{24}$ For the gold atoms lying on top of the semi-infinite substrate (e.g. describing the atomicscale structures of the surface as discussed in sec. II), we use the standard double-zeta molecular basis set as described by P. J. Hay and W. R. Wadt (J. Chem. Phys. 82, 270 (1985)); For the gold atoms on the surface of the semi-infinite substrate, we follow the standard convention in surface and solid state electronic structure calculations using molecular Gaussian-type orbital basis sets to construct a minimum valence basis set by removing the most diffused Gaussian primitive from the double-zeta contraction. See footnotes 52 and 77 of paper $\mathbf{I}$. 
${ }^{25}$ GAUSSIAN 98, Revision A.7, M. J. Frisch, G. W. Trucks, H. B. Schlegel, G. E. Scuseria, M. A. Robb, J. R. Cheeseman, V. G. Zakrzewski, J. A. Montgomery Jr., R. E. Stratmann, J. C. Burant, S. Dapprich, J. M. Millam, A. D. Daniels, K. N. Kudin, M. C. Strain, O. Farkas, J. Tomasi, V. Barone, M. Cossi, R. Cammi, B. Mennucci, C. Pomelli, C. Adamo, S. Clifford, J. Ochterski, G. A. Petersson, P. Y. Ayala, Q. Cui, K. Morokuma, D. K. Malick, A. D. Rabuck, K. Raghavachari, J. B. Foresman, J. Cioslowski, J. V. Ortiz, A. G. Baboul, B. B. Stefanov, G. Liu, A. Liashenko, P. Piskorz, I. Komaromi, R. Gomperts, R. L. Martin, D. J. Fox, T. Keith, M. A. Al-Laham, C. Y. Peng, A. Nanayakkara, C. Gonzalez, M. Challacombe, P. M. W. Gill, B. Johnson, W. Chen, M. W. Wong, J. L. Andres, C. Gonzalez, M. Head-Gordon, E. S. Replogle and J. A. Pople", Gaussian, Inc. , Pittsburgh, PA, 1998.

${ }^{26}$ B. C. Stipe, M. A. Rezaei and W. Ho, Science 280, 1732 (1998); Phys. Rev. Lett. 82, 1724 (1999).

${ }^{27}$ M. Di Ventra, S. T. Pantelides and N. D. Lang, Phys. Rev. Lett. 84, 979 (2000).

${ }^{28}$ W. Tian, S. Datta, S. Hong, R. Reifenberger, J. J. Henderson and C. P. Kubiak, J. Chem. Phys. 109, 2874 (1998).

${ }^{29}$ M. Tsukada et al. , Surf. Sci. Rep. 13, 265 (1991).

${ }^{30}$ N.D. Lang, Phys. Rev. B 55, 9364 (1997).

${ }^{31}$ I. -W. Lyo and Ph. Avouris, Science 245, 1369 (1989).

${ }^{32}$ S.N. Yaliraki and M.A. Ratner, J. Chem. Phys. 109, 5036 (1998). 


\section{FIGURES}

FIG. 1. Transmission versus energy (TE) and projected density of states (PDOS) in units of $(1 / \mathrm{eV})$ corresponding to the HOMO and LUMO as a function of the top metal-molecule distance $\Delta L$ at $\Delta L=0.5,1.0(\AA)$. For comparison, we have also shown the TE and PDOS characteristics for the reference device structure where the molecule forms symmetric contact with the two electrodes (solid line). The horizontal line in the TE plot shows the Fermi-level position. The horizontal lines in the PDOS plot show the energies of the HOMO and LUMO levels in the isolated molecule.

FIG. 2. Transmission versus energy (TE) and projected density of states (PDOS) in units of $(1 / \mathrm{eV})$ corresponding to the HOMO and LUMO as a function of the top metal-molecule distance $\Delta L$ at $\Delta L=1.5,2.0(\stackrel{\circ}{A})$. For comparison, we have also shown the TE and PDOS characteristics for the reference device structure where the molecule forms symmetric contact with the two electrodes (solid line). The horizontal line in the TE plot shows the Fermi-level position. The horizontal lines in the PDOS plot show the energies of the HOMO and LUMO levels in the isolated molecule.

FIG. 3. Current-voltage (I-V) and conductance-voltage (G-V) characteristics of the gold-PDT-gold junction as a function of the top metal-molecule distance. For comparison, we have also shown the I-V and G-V characteristics for the reference device structure where the molecule forms symmetric contact with the two electrodes (solid line).

FIG. 4. Bias-induced modification of molecular levels as a function of top metal-molecule distance. We have also shown the position of the equilibrium Fermi-level $E_{F}$ and the electrochemical potential of the two electrodes $\mu_{L(R)}$ in the plot.

FIG. 5. Spatial distribution of charge transfer and potential drop at bias voltage of $2.0(\mathrm{~V})$ at the gold-PDT-gold contact for $\Delta=1.5(\AA)$. The upper figure shows the difference between the electron density at finite bias voltage and the electron density for device at equilibrium, the lower figure shows the difference between the electrostatic potential at that voltage and the electrostatic potential for device at equilibrium. 
FIG. 6. Three dimensional plot of the bias-dependence of the transmission versus energy characteristics for $\Delta=1.5(\AA)$. The two lines in the $\mathrm{X}-\mathrm{Y}$ plane show the electrochemical potential of the two electrode $\mu_{L(R)}$ as a function of applied bias voltage.

FIG. 7. Transmission versus energy (TE) and projected density of states (PDOS) in units of $(1 / \mathrm{eV})$ corresponding to the HOMO-1, HOMO, LUMO and LUMO+1 for device model 1 and model 2. The horizontal line in the TE plot shows the Fermi-level position. The horizontal lines in the PDOS plot show the energies of the HOMO-1, HOMO, LUMO and LUMO+1 levels in the isolated molecule.

FIG. 8. Current-voltage (I-V) and conductance-voltage (G-V) characteristics of the gold-PDT-gold for device model 1 and 2 .

FIG. 9. Electrostatic potential change upon the formation of the molecular junction for model 1 as a function of position in the xy-plane. Also shown is the projection of the molecule and the apex atom onto the xy-plane.

FIG. 10. Spatial distribution of charge transfer and potential drop at bias voltage of $-2.0(V)$ and $2.0(V)$ for device model 1.

FIG. 11. Transmission versus energy (TE) and projected density of states (PDOS) in units of (1/eV) corresponding to the HOMO and LUMO for the atop adsorption geometry with symmetric contact (model 3) and with increased top metal-molecule distance (model $4, \Delta L=1.0(\AA)$ ).

FIG. 12. Current-voltage (I-V) and conductance-voltage (G-V) characteristics of the gold-PDT-gold junction as a function of molecular adsorption geometry.

FIG. 13. Orbital shape of the HOMO-1, HOMO and LUMO states of PDT molecule with an additional hydrogen end atom for both spin-up and spin-down electrons. 
FIG. 14. Transmission versus energy (TE) and projected density of states (PDOS) in units of $(1 / \mathrm{eV})$ corresponding to the HOMO-1, HOMO, LUMO and LUMO+1 for the molecule with the end hydrogen atom. The horizontal line in the TE plot shows the Fermi-level position. The horizontal lines in the PDOS plot show the energies of the HOMO and LUMO levels for spin-up electrons in the isolated molecule.

FIG. 15. Current-voltage (I-V) and conductance-voltage (G-V) characteristics for the molecule with the end hydrogen atom.

FIG. 16. Three dimensional plot of the bias-dependence of the transmission versus energy characteristics for the molecule with the end hydrogen atom. The two lines in the X-Y plane show the electrochemical potential of the two electrode $\mu_{L(R)}$ as a function of applied bias voltage. 
This figure "xueFig2-1_1.jpg" is available in "jpg" format from: http://arxiv.org/ps/cond-mat/0303180v1 
This figure "xueFig3-1.jpg" is available in "jpg" format from: http://arxiv.org/ps/cond-mat/0303180v1 
This figure "xueFig4-1.jpg" is available in "jpg" format from: http://arxiv.org/ps/cond-mat/0303180v1 
This figure "xueFig5-1.jpg" is available in "jpg" format from: http://arxiv.org/ps/cond-mat/0303180v1 
This figure "xueFig2-1_2.jpg" is available in "jpg" format from: http://arxiv.org/ps/cond-mat/0303180v1 
This figure "xueFig2-2.jpg" is available in "jpg" format from: http://arxiv.org/ps/cond-mat/0303180v1 
This figure "xueFig3-2.jpg" is available in "jpg" format from: http://arxiv.org/ps/cond-mat/0303180v1 
This figure "xueFig4-2.jpg" is available in "jpg" format from: http://arxiv.org/ps/cond-mat/0303180v1 
This figure "xueFig5-2.jpg" is available in "jpg" format from: http://arxiv.org/ps/cond-mat/0303180v1 
This figure "xueFig2-3.jpg" is available in "jpg" format from: http://arxiv.org/ps/cond-mat/0303180v1 
This figure "xueFig3-3.jpg" is available in "jpg" format from: http://arxiv.org/ps/cond-mat/0303180v1 
This figure "xueFig5-3.jpg" is available in "jpg" format from: http://arxiv.org/ps/cond-mat/0303180v1 
This figure "xueFig2-4.jpg" is available in "jpg" format from: http://arxiv.org/ps/cond-mat/0303180v1 
This figure "xueFig3-4.jpg" is available in "jpg" format from: http://arxiv.org/ps/cond-mat/0303180v1 
This figure "xueFig5-4.jpg" is available in "jpg" format from: http://arxiv.org/ps/cond-mat/0303180v1 
This figure "xueFig2-5.jpg" is available in "jpg" format from: http://arxiv.org/ps/cond-mat/0303180v1 